\title{
Dégradabilité de l'herbe ingérée par des chèvres au pâturage au cours de deux saisons
}

\author{
V Fedele, R Rubino, S Claps \\ Istituto Sperimentale per la Zootecnia, Viale Basento, 106, 85100 Potenza, Italie
}

Jusqu'à maintenant la cinétique de dégradation ruminale des espèces végétales prises individuellement a été peu étudiée et celle de l'ensemble sélectionné par la chèvre ne l'a pas été du tout. Pour l'étudier on a prélevé le bol alimentaire de 3 chèvres portant des fistules oesophagiennes qui pâturaient deux types de prairies, l'une en fond de vallée (Bella, $330 \mathrm{~m}$ d'altitude), l'autre en montagne (Li Foy, $1250 \mathrm{~m}$ d'altitude), et à deux saisons différentes : au printemps et en été. Les bols, après mélange et lavage avec de l'eau distillée contenant $2 \%$ d'acide acétique, ont été séchés puis broyés a travers une grille de $2,5 \mathrm{~mm}$. $30 \mathrm{~g}$ d'échantillon ont été placés en sac de Nylon et mis à incuber à 7 h00 du matin pendant 24 heures dans le rumen de 2 chères portant des fistules ruminales. Les chèvres ont été au pâturage sur une prairie naturelle après le premier prélèvement, jusqu'à 7 h00 du soir (12 heures). Elles ont été, ensuite, alimentées en chèvrerie avec la même herbe du pâturage. Des prélèvements de jus de rumen ont été effectués après $2,4,6,8,12$ et 24 heures la pose des sachets. Le $\mathrm{pH}$ et la teneur en $\mathrm{NH}_{3}$ ont été déterminés.

La dégradation des matières azotées (MAT) au temps zéro, c'est-à-dire la fraction immédiatement dégradable a été plus élevée au printemps (environ $40 \%$ ) et plus faible en été où une différence significative est apparue entre les deux sites (31,4 de Bella vs 21,3 de Li Foy). Cela s'explique par une composition floristique différente du pâturage qui entraîne certainement des variations de la nature des MAT récoltées à Li Foy. Les bols y avaient une proportion en légumineuses plus élevée que ceux de Bella surtout en été où le contenu en MAT du bol est plus élevé. Cependant, la dégradation à $24 \mathrm{~h}$ a été significativement plus élevée à Li Foy d'environ 8 points en moyenne. La teneur en $\mathrm{NH}_{3}$ a été, en général, supérieure à $15 \mathrm{mg} / 100 \mathrm{ml}$ et a atteint le maximum après 8 heures de séjour des chèvres sur la prairie. Cela est conforme aux résultats de Martillotti et al (1994, Ann Zootech, 43, (3), 241) obtenus sur les vaches au pâturage. Les valeurs de $\mathrm{NH}_{3}$ pendant l'été à Bella sont nettement plus faibles qu'à Li Foy ; ce qui peut être en relation avec la faible disponibilité en MAT des fourrages ingérés.

Le $\mathrm{pH}$, en général, diminue jusqu'à 12 heures atteignant le minimum de 5,75 au printemps à $\mathrm{Li}$ Foy et ensuite remonte. II n'est pas influencé par l'augmentation du $\mathrm{NH}_{3}$ puisqu'il n'y a pas de corrélation entre les deux paramètres.

En conclusion, la saison et l'altitude se répercutent sur la composition floristique et les stades phénologiques de la plante. En conséquence, elles influencent fortement les dégradabilités des plantes ingérées par les chèvres au pâturage.

\begin{tabular}{|c|c|c|c|c|c|c|c|c|c|c|c|c|}
\hline \multirow{3}{*}{$\begin{array}{l}\text { Heures } \\
\text { de } \\
\text { prélève- } \\
\text { ment }\end{array}$} & \multicolumn{6}{|c|}{ Li Foy $(1250 \mathrm{~m}, \mathrm{~s} / \mathrm{m})$} & \multicolumn{6}{|c|}{ Bella $(330 \mathrm{~m}, \mathrm{~s} / \mathrm{m})$} \\
\hline & \multicolumn{3}{|c|}{ Printemps MAT (\%) 11,1} & \multicolumn{3}{|c|}{ Eté MAT $(\%) 16,4$} & \multicolumn{3}{|c|}{ Printemps MAT (\%) 11,2} & \multicolumn{3}{|c|}{ Eté MAT (\%) 9,2 } \\
\hline & MAT & $\mathrm{NH}_{3}$ & $\mathrm{pH}$ & MA & $\mathrm{NH}_{3}$ & $\mathrm{pH}$ & $N$ & $\mathrm{NH}_{3}$ & & MA & $\mathrm{NH}_{3}$ & $\mathrm{pH}$ \\
\hline & & 768 & & & & & & & & & & \\
\hline & & $15,96^{\mathrm{Bc}}$ & 6,78 & $4 \mathrm{Be}$ & 21, & $6,00^{\mathrm{Bb}}$ & & $2^{\mathrm{Aa}}$ & & $33,7^{\mathrm{Bd}}$ & $6^{\mathrm{Bb}}$ & \\
\hline & Ac & $20,48^{\mathrm{Aa}}$ & $6,09 \mathrm{ABCb}$ & $40,0^{\mathrm{Cd}}$ & & $5,95^{\mathrm{Bb}}$ & & $22,92^{\mathrm{Aa}}$ & & $37,6^{\mathrm{CC}}$ & $6,68^{B C}$ & 5,8 \\
\hline & $3,0^{A C}$ & $21,08^{\text {Aa }}$ & & $49,0^{B C C}$ & & $6,23^{A b}$ & & & & $46,2^{\mathrm{Cb}}$ & $8^{\mathrm{Bb}}$ & \\
\hline 12 & $8,7^{\mathrm{Ab}}$ & $19,88^{\mathrm{Aab}}$ & & $55,3^{A b}$ & $16,04^{\text {Ad }}$ & $6,08^{A B b}$ & & $9,52^{\mathrm{Bc}}$ & $3 \mathrm{AC}$ & $56,0^{\mathrm{Aa}}$ & $11,84 \mathrm{Bb}$ & $5,90^{\mathrm{ABt}}$ \\
\hline 24 & $66,7^{\text {Ba }}$ & $9,88^{\mathrm{Bd}}$ & $7,04 \mathrm{Aa}$ & $76,9^{\mathrm{Aa}}$ & $22,92^{\mathrm{Aa}}$ & $6,84^{A a}$ & $62,9 \mathrm{Ca}$ & $11,00^{\mathrm{BC}}$ & $6,94^{\mathrm{Aab}}$ & $64,9 \mathrm{BC}$ & $16,482^{\mathrm{Aa}}$ & $6,92^{\mathrm{Aa}}$ \\
\hline
\end{tabular}

lettres majuscules valeur $P \leq 0,05$ sur lignes

lettres minuscules valeur $\mathrm{P} \leq 0,05$ sur colonnes 